\title{
Exercise Training in Patients Receiving Maintenance Hemodialysis: A Systematic Review of Clinical Trials
}

\author{
Birinder Singh B. Cheema ${ }^{a}$ Maria A. Fiatarone Singh ${ }^{a, b, c}$ \\ a School of Exercise and Sport Science, and ${ }^{b}$ Faculty of Medicine, University of Sydney, Sydney, Australia; \\ ${ }^{c}$ Hebrew Rehabilitation Center for the Aged and Jean Mayer USDA Human Nutrition Center on Aging, \\ Tufts University, Boston, Mass., USA
}

\section{Key Words}

Aerobic training $\cdot$ Resistance training $\cdot$ End-stage renal disease $\cdot$ Quality of life $\cdot$ Health

\begin{abstract}
Background: Exercise is not routinely advocated in patients with end-stage renal disease (ESRD) receiving maintenance hemodialysis (HD), compared to best practice in other chronically diseased cohorts. Lack of widespread awareness of the exercise in HD literature may be contributing to these shortcomings of clinical practice. Therefore, our objectives are: (1) to systematically review trials of exercise training involving adult HD patients; (2) to provide empirical evidence that exercise can elicit health-related adaptations in this cohort, and (3) to provide recommendations for future investigations. Method: A systematic review of the literature using computerized databases was performed. Results: According to the 29 trials reviewed, HD patients can safely derive a myriad of health-related adaptations from engaging in
\end{abstract}

B. Cheema is a PhD research student enrolled at the School of Exercise and Sport Science, University of Sydney, Australia.

\section{KARGER}

Fax +41613061234 E-Mail karger@karger.ch www.karger.com
(C) 2005 S. Karger AG, Basel

0250-8095/05/0254-0352\$22.00/0

Accessible online at:

www.karger.com/ajn appropriately structured exercise regimens involving aerobic and/or resistance training. However, methodological limitations within this body of literature may be partially responsible for minimal advocacy for exercise in this cohort. Conclusions: Robustly designed RCTs with thorough, standardized reporting are required if clinical practice and quality of life of this cohort is to be enhanced through the integration of exercise training and mainstream medical practice. Future trials should demonstrate the clinical importance, and long-term feasibility and applicability of exercise training for this vulnerable patient population.

Copyright $\odot 2005$ S. Karger AG, Basel

\section{Introduction}

According to the United States Renal Data System (USRDS), the incidence of end-stage renal disease (ESRD) continues to increase each year [1]. Over 100,000 new cases were reported in the USRDS 2004 report [1] and over 430,000 individuals in the United States currently live with ESRD [1]. Rising incidence and prevalence trends are being reported in many other countries maintaining renal registries [2]. 
Approximately $91.9 \%$ of patients diagnosed with ESRD receive maintenance hemodialysis (HD) treatment as renal replacement therapy [1]. This intervention is typically prescribed 3 times per week, $4-6 \mathrm{~h}$ per session, and remains ongoing for the lifetime of the patient or until successful kidney transplantation. Although advances in HD treatment have extended the lifespan of patients with ESRD, this treatment alone does not ensure preservation of quality of life (QOL). Hemodialysis patients typically suffer from significant impairments of QOL as compared to their healthy counterparts, or those with successful kidney transplants.

Planned exercise, involving aerobic and resistance training modalities, has become well-recognized as a therapeutic intervention that can ameliorate the marked physiological, functional, and psychological deterioration which commonly accrues as a consequence of biological aging, catabolic illness, and a sedentary lifestyle, factors that may all contribute to the progressive decline of vitality and QOL commonly observed in HD patients. As such, many trials of exercise training have been conducted with HD patients over the past 3 decades. Findings from virtually all of these trials have demonstrated that prolonged exercise is safe and beneficial for this patient population. However, recent evidence has clearly suggested that exercise is still not routinely advocated or prescribed in this cohort $[3,4]$, compared to best practice in other diseased populations, such as those with cardiac and pulmonary disease. The lack of widespread awareness of the exercise in the HD literature may potentially be contributing to the shortcomings of clinical practice with regard to prescribing exercise.

Therefore our objectives are:

(1) To systematically review trials of exercise training involving adult hemodialysis patients.

(2) To provide empirical evidence that exercise can counteract the marked physiological, functional, and psychological wasting associated with ESRD.

(3) To provide recommendations for future investigations which may potentially lead to the integration of exercise prescription within the mainstream of medical practice for this patient population.

\section{Method}

A systematic, critical review rather than a meta-analytic approach has been taken as the heterogeneity of exercise modalities and dosages utilized and outcomes assessed do not lend themselves to meta-analytic methods. There are also clinically important results to discuss in some of the uncontrolled trials.
Criteria for Considering Studies

Study Designs

Randomized controlled trials (RCTs), controlled trials and uncontrolled trials were included. Abstracts and case reports were not considered.

Subjects

Subjects were adult ( $\geq 18$ years) men and women receiving HD treatment for the management of ESRD. Studies involving young ( $<18$ years) HD patients, continuous ambulatory peritoneal dialysis patients and/or pre-dialysis patients were not considered.

Interventions

Trials prescribing aerobic and/or resistance training modalities $\geq 5$ weeks in duration were included. Studies investigating the effects of single, acute bouts of exercise, or studies applying interventions $<5$ weeks were excluded. Studies involving multimodal interventions (e.g. exercise combined with nutritional supplementation) were also excluded.

Outcome Measures

Outcome measures potentially responsive to exercise training, based on evidence of exercise training in other diseased and nondiseased cohorts, were considered. These outcomes included a broad spectrum of physiological, psychological, and functional measures.

\section{Search Method}

We conducted a literature review in November 2004 from the years 1966 to 2004, limited to the English language, using computerized databases, including Medline, CINAHL, SportDiscus, Embase, and Web of Science. The search combined key words related to HD treatment (i.e. hemodialysis, haemodialysis, dialysis, renal replacement therapy), ESRD (i.e. nephrology, nephron, kidney, renal disease, renal failure) and exercise (i.e. exercise, training, physical activity, rehabilitation, resistance training, aerobic training, strength training, muscle, endurance, $\mathrm{VO}_{2 \text { peak }}$ ). Articles retrieved were examined for further relevant references.

\section{Results of Search}

\section{Study Designs and Research Quality}

The search resulted in 34 articles presenting the findings of 29 trials, including 9 uncontrolled trials $(9 / 29$, $31 \%)$ [5-15], 7 controlled trials $(7 / 29,24 \%)$ [16-23], and 13 RCTs $(13 / 29,45 \%)$ [24-38].

All 9 uncontrolled trials [5-15] involved time series investigation of a single treatment group evaluated with repeated measures collected before and after training (table 1). One uncontrolled trial utilized the noncompliant subjects as a comparison group for statistical analyses [7]; however, the study was originally intended as a single treatment group design. None of the 9 uncontrolled trials mentioned the involvement of blinded outcomes assessors. 
Table 1. Uncontrolled trials of exercise training in hemodialysis

\begin{tabular}{|c|c|c|c|c|c|c|c|}
\hline \multirow{2}{*}{$\begin{array}{l}\text { Authors (year) } \\
\text { country }\end{array}$} & \multirow[t]{2}{*}{$\mathrm{n}$} & \multirow{2}{*}{$\begin{array}{l}\text { Study } \\
\text { groups }\end{array}$} & \multicolumn{4}{|c|}{ Exercise intervention } & \multirow{2}{*}{$\frac{\text { Outcomes }}{\text { variable }}$} \\
\hline & & & delivery & modality & prescription & duration & \\
\hline $\begin{array}{l}\text { Goldberg et al. [5] } \\
\text { (1979) } \\
\text { Goldberg et al. [6] } \\
\text { (1980) USA }\end{array}$ & 7 & $\begin{array}{l}\text { exercise } \\
(\mathrm{n}=7)\end{array}$ & NDT & AER & $\begin{array}{l}\text { cycle erg., walking, } \\
\text { jogging, calisthenics } \\
65-75 \% \mathrm{HR}_{\max } \\
\text { to tolerance }\end{array}$ & 8-9 months & $\begin{array}{l}\text { plasma triglyceride } \\
\text { VLDL triglygeride } \\
\text { HDL cholesterol } \\
\text { fasting plasma glucose } \\
\text { glucose disappearance rate } \\
\text { fasting plasma insulin } \\
\text { hematocrit } \\
\text { hemoglobin } \\
\mathrm{VO}_{2 \text { peak }} \\
\text { GXT duration }\end{array}$ \\
\hline
\end{tabular}

\begin{tabular}{|c|c|c|c|c|c|c|c|c|c|}
\hline $\begin{array}{l}\text { Shalom et al. [7] } \\
\text { (1984) USA }\end{array}$ & 14 & $\begin{array}{l}\text { exercise } \\
(n=14)\end{array}$ & NDT & AER & $\begin{array}{l}\text { cycle erg., calisthenics, } \\
\text { walking jogging } \\
\text { up to } 75-80 \% \mathrm{HR}_{\max } \\
45 \mathrm{~min}, 5 \times / \text { week }\end{array}$ & 12 weeks & $\begin{array}{l}\text { GXT workload } \\
\mathrm{VO}_{2 \text { peak }} \\
\text { oxygen pulse }\end{array}$ & $\begin{array}{l}\text { increase } \\
\text { increase } \\
\text { increase }\end{array}$ & $\begin{array}{l}0.007 \\
0.001 \\
0.026\end{array}$ \\
\hline $\begin{array}{l}\text { Moore et al. [8] } \\
\text { (1993) USA }\end{array}$ & 23 & $\begin{array}{l}\text { exercise } \\
(n=23)\end{array}$ & ID & AER & $\begin{array}{l}\text { cycle erg. training, } \\
\text { RPE } 6 / 10 \\
\leq 60 \mathrm{~min}, 3 \times / \text { week }\end{array}$ & 12 weeks & $\begin{array}{l}\text { maximal workload } \\
\text { submaximal heart rate } \\
\text { phosphofructokinase activity } \\
\text { type I muscle fibre area } \\
\text { type II muscle fiber area } \\
\text { capillary to muscle fibre ratio }\end{array}$ & $\begin{array}{l}\text { increase } \\
\text { decrease } \\
\text { increase } \\
\text { no } \Delta \\
\text { no } \Delta \\
\text { no } \Delta\end{array}$ & $\begin{array}{l}\leq 0.05 \\
\leq 0.05 \\
\leq 0.05 \\
- \\
- \\
-\end{array}$ \\
\hline $\begin{array}{l}\text { Kouidi et al. [9] } \\
\text { (1998) Greece }\end{array}$ & 7 & $\begin{array}{l}\text { exercise } \\
(n=7)\end{array}$ & NDT & COMBO & $\begin{array}{l}\text { aerobic and strength } \\
\text { training, } \\
90 \mathrm{~min}, 3 \times / \text { week }\end{array}$ & 6 months & $\begin{array}{l}\text { GXT duration } \\
\mathrm{VO}_{2 \text { peak }} \\
\text { peak blood lactate } \\
\text { nerve conduction velocity } \\
\text { isometric strength (LB) } \\
\text { type } 1 \text { muscle fibre area } \\
\text { type II muscle fibre area } \\
\text { percentage of type II fibres } \\
\text { mean muscle fibre area }\end{array}$ & $\begin{array}{l}\text { increase } \\
\text { increase } \\
\text { decrease } \\
\text { increase } \\
\text { increase } \\
\text { increase } \\
\text { increase } \\
+23.0 \% \\
\text { increase }\end{array}$ & $\begin{array}{l}\leq 0.05 \\
\leq 0.05 \\
\leq 0.05 \\
\leq 0.05 \\
\leq 0.05 \\
\leq 0.05 \\
\leq 0.05 \\
\leq 0.05 \\
\leq 0.05\end{array}$ \\
\hline $\begin{array}{l}\text { Ridley et al. [10] } \\
\text { (1999) Canada }\end{array}$ & 8 & $\begin{array}{l}\text { exercise } \\
(n=8)\end{array}$ & ID & COMBO & $\begin{array}{l}\text { cycle ergometer, } \\
\leq 30 \text { min, } \\
\text { to tolerance and } \\
6 \text { strength exercises up } \\
\text { to } 30 \text { reps, } 2 \times / \text { week }\end{array}$ & 12 weeks & $\begin{array}{l}\text { 6-minute walk distance } \\
\text { resistance training load } \\
\text { Piper Fatigue Scale: } \\
\text { affective fatigue } \\
\text { sensory scale fatigue }\end{array}$ & $\begin{array}{l}\text { increase } \\
\text { increase } \\
\text { decrease } \\
\text { decrease }\end{array}$ & $\begin{array}{l}\leq 0.006 \\
\leq 0.013 \\
\leq 0.05 \\
\leq 0.03\end{array}$ \\
\hline $\begin{array}{l}\text { Zaluska et al. [11] } \\
\text { (2002) Poland }\end{array}$ & 10 & $\begin{array}{l}\text { exercise } \\
(n=10)\end{array}$ & ID & AER & $\begin{array}{l}\text { cycle ergometer } \\
30 \mathrm{~min}, 3 \times / \text { week }\end{array}$ & 6 months & $\begin{array}{l}\text { albumin } \\
\text { C-reactive protein } \\
\text { protein catabolic rate } \\
\mathrm{Kt} / \mathrm{V}\end{array}$ & $\begin{array}{l}\text { increase } \\
\text { decrease } \\
\text { increase } \\
\text { increase }\end{array}$ & $\begin{array}{l}\leq 0.024 \\
\leq 0.046 \\
\leq 0.001 \\
\leq 0.026\end{array}$ \\
\hline $\begin{array}{l}\text { Oh-Park et al. [12] } \\
\text { (2002) USA }\end{array}$ & 22 & $\begin{array}{l}\text { exercise } \\
(n=22)\end{array}$ & ID & СОМBO & $\begin{array}{l}\text { cycle ergometer } \\
\text { up to } 30 \text { min } \\
\text { to tolerance } \& \text { knee } \\
\text { extensions } \\
3 \text { sets } \times 15 \text { reps, } \\
50 \% 1 \mathrm{RM}, 2-3 \times / \text { week }\end{array}$ & 3 months & $\begin{array}{l}\text { knee extension strength } \\
\text { SF-36 - physical functioning } \\
\text { SF-36 - mental health }\end{array}$ & $\begin{array}{l}\text { increase } \\
\text { increase } \\
\text { increase }\end{array}$ & $\begin{array}{l}\leq 0.0001 \\
\leq 0.003 \\
\leq 0.0004\end{array}$ \\
\hline $\begin{array}{l}\text { Headley et al. [13] } \\
(2002) \\
\text { Nindl et al. [14] } \\
\text { (2004) USA }\end{array}$ & 10 & $\begin{array}{l}\text { exercise } \\
(n=10)\end{array}$ & $\begin{array}{l}\text { NDT\& } \\
\mathrm{HB}\end{array}$ & PRT & $\begin{array}{l}\text { machine weight } \\
\text { exercises }(8-9) \text {, } \\
1-2 \text { sets } \times 15 \text { reps } \\
2 \times / \text { week and } \\
\text { at week } 7: 9 \text { home- } \\
\text { based PRT exercises } \\
\text { using Theraband }{ }^{\mathrm{TM}} \\
1 \times / \text { week }\end{array}$ & 12 weeks & $\begin{array}{l}\text { peak isometric force at } 90^{\circ} \\
6 \text {-minute walk distance } \\
\text { maximal walking speed } \\
\text { sit-to-stand time } x 10 \text { reps } \\
\text { percentage body fat } \\
\text { C-reactive protein } \\
\text { total IGF-1 } \\
\text { IGF-1:IGFBP-3 }(\times 10) \text { ratio }\end{array}$ & $\begin{array}{l}\text { increase } \\
\text { increase } \\
\text { increase } \\
\text { increase } \\
\text { increase } \\
\text { decrease } \\
\text { decrease } \\
\text { decrease }\end{array}$ & $\begin{array}{l}\leq 0.05 \\
\leq 0.05 \\
\leq 0.05 \\
\leq 0.05 \\
\leq 0.05 \\
\mathrm{NR} \\
\leq 0.039 \\
\leq 0.003\end{array}$ \\
\hline $\begin{array}{l}\text { Mustata et al. [15] } \\
\text { (2004) Canada }\end{array}$ & 11 & $\begin{array}{l}\text { exercise } \\
(\mathrm{n}=11)\end{array}$ & NDT & AER & $\begin{array}{l}\text { treadmill walking or } \\
\text { cycle ergometer } \\
40-50 \text { min, } 2 \times / \text { week }\end{array}$ & 3 months & $\begin{array}{l}\text { arterial stiffness } \\
\text { pulse pressure }(\mathrm{mm} \mathrm{Hg}) \\
\text { SBP } \\
\text { insulin resistance }\end{array}$ & $\begin{array}{l}\text { decrease } \\
\text { decrease } \\
\text { decrease } \\
\text { no } \Delta\end{array}$ & $\begin{array}{r}0.01 \\
<0.05 \\
<0.05\end{array}$ \\
\hline
\end{tabular}

NDT = Nondialysis time; ID = intradialytic; $\mathrm{HB}=$ home based; AER = aerobic training; COMBO = aerobic plus lower-intensity strength training; PRT = progressive resistance training; GXT = graded exercise test; $\mathrm{RPE}=$ rating of perceived exertion; $\mathrm{HR}_{\max }=$ maximal heart rate; $\mathrm{SBP}=$ systolic blood pressure; $\mathrm{DBP}=$ diastolic blood pressure; NR = not reported.

All $\mathrm{p}$ values calculated by comparing within group change over time (i.e. pretest - posttest). 
Seven controlled trials [16-23] involved a treatment (i.e. exercise) and a control (i.e. nonexercise) group, consisting of HD patients but without random assignment of subjects into these two groups (table 2). Statistical analyses performed in $4 / 7$ trials (57\%) involved repeated measures comparisons within groups only, while 3/7 trials (43\%) [20-22, 27] performed statistical comparisons between groups. None of the 7 controlled trials mentioned the involvement of blinded outcomes assessors.

Thirteen trials involved randomization of subjects (table 3) [24-38]. Nine RCTs (9/13, 69\%) assigned subjects to a treatment or nontreatment control group [24-26, 28$32,35,37,38]$. Three additional RCTs $(3 / 13,23 \%)$ randomized subjects to: (a) intradialytic vs. non-dialysis exercise interventions [36]; (b) exercise vs. sham exercise (i.e. placebo) [33], or (c) exercise vs. a social support group [27]. One study $(1 / 13,8 \%)$ compared the effects of exercise training plus the normalization of blood hematocrit within a 4-group RCT [34]. Eleven RCTs (11/13, 85\%) performed between group comparisons on repeated measures [27, 29-38], while 2 trials $(2 / 13,15 \%)$ [24-26, 28] reported change over time within groups only. Six RCTs $(6 / 13,46 \%)[24-26,29,31,35-37]$ reported that randomization of subjects occurred following baseline testing. Only two RCTs $(2 / 13,15 \%)$ reported that partial or complete collection of outcome measures were performed by blinded assessors [29, 33]. To date, only two RCTs $(2 / 13$, $15 \%)$ involved intention-to-treat strategy of analysis $[33,34]$.

\section{Overview of the Subjects}

Sample Sizes

Nine hundred and fifty-nine $(\mathrm{n}=959)$ patients have been enrolled in the 29 trials reviewed. Sample size ranged from 7 to 286 enrolled patients. Thirteen trials (13/29, $45 \%)$ have enrolled $<20$ patients, 15 trials $(15 / 29,52 \%)$ have enrolled $20-75$ patients, and only one trial $(3 / 29$, $3 \%)$ has enrolled $>75$ patients $(n=286)($ tables $1-3)[20$, 21].

\section{Gender}

Excluding two trials that did not provide a gender breakdown (where combined $n=20$ ) $[11,16], 479$ men and 460 women (51:49) have been enrolled in the trials reviewed. Except for one trial involving 12 men only [17], all trials included men and women.

\section{Age}

Age of the sample was expressed as mean \pm SD in 10 trials $(10 / 29,34 \%)[18-23,27,28,33,34,38]$ in which mean age ranged from $36 \pm 3$ to $60 \pm 17$ [27, 38]. In 18 trials $(18 / 29,62 \%)$ that presented an age range [5-10, 12-17, 24-26, 29-32, 35-37], the youngest and eldest patients enrolled were 19 years [24-26] and 84 years [12], respectively. A broad age range was generally reported. One trial $(1 / 29,3 \%)$ did not describe the age of their sample [11].

\section{Duration of Hemodialysis}

Entry criteria typically precluded patients receiving HD treatment $<3$ months. Length of HD treatment ranged from 0.25 to 17.4 years $[13,14]$ in trials providing these data. Three trials $(3 / 29,10 \%)$ did not delimit an entry criterion, or describe their sample with respect to this factor [10-12].

\section{Etiology of Renal Failure}

Sixteen trials $(16 / 29,55 \%)$ detailed the etiology of renal failure in their sample [5-9, 12-15, 20-22, 24-28, 33, 34, 37, 38]. Common causes of ESRD in these trials included glomerulonephritis (32\%), hypertension (18.5\%), and diabetes (12\%). Diabetes was a prevalent predisposing factor despite the fact that $5 / 16$ trials $(31 \%)$ that described ESRD etiology excluded diabetics [7, 9, 24-27, 37].

\section{Comorbidities}

Eleven trials provided information regarding common comorbidities in their sample $[5-8,10,17,22,31,32,35$, $36,38]$. The average prevalence of common comorbidities among these trials included hypertension (71\%), cardiovascular disease (34\%), and diabetes (21\%). Diabetes was prevalent in $4 / 29$ trials (14\%) providing these data [17, 22, 33, 38]. However, at least $11 / 29$ trials to date (34\%), including 9/13 RCTs (69\%), have excluded patients with diabetes [7, 9, 26-29, 31-34, 37, 40, 41]. Other trials have excluded patients with ischemic heart disease [8, 33, 34], and congestive heart failure [10, 24-27, 36].

\section{Overview of the Exercise Interventions \\ Duration}

Duration ranged from 6 weeks [30] to 4 years [36]. The majority of interventions extended for 3-6 months [8-16, $18-22,27-29,31-35,37]$, with 3 trials of shorter duration $[23,30,38]$ and 4 trials of longer duration $[5,6,17$, 24-26, 36]. Kouidi et al. [36] have conducted the longest trial to date (4 years).

Am J Nephrol 2005;25:352-364 
Table 2. Nonrandomized controlled trials of exercise training in hemodialysis

\begin{tabular}{|c|c|c|c|c|c|c|c|c|c|}
\hline \multirow{2}{*}{$\begin{array}{l}\text { Authors (year) } \\
\text { country }\end{array}$} & \multirow[t]{2}{*}{$\mathrm{n}$} & \multirow[t]{2}{*}{ Study groups } & \multicolumn{4}{|c|}{ Exercise intervention } & \multicolumn{3}{|l|}{ Outcomes } \\
\hline & & & delivery & modality & prescription & duration & variable & $\%$ change & $\mathrm{p}$ value \\
\hline $\begin{array}{l}\text { Zabetakis et al. } \\
{[16](1982)} \\
\text { USA }\end{array}$ & 10 & $\begin{array}{l}\text { exercise }(n=5) \\
\text { control }(n=5)\end{array}$ & NDT & AER & $\begin{array}{l}\text { treadmill walking/ } \\
\text { jogging at AT, } \\
25-45 \text { min, } 3 \times / \text { week }\end{array}$ & 10 weeks & $\begin{array}{l}\text { after } 5 \text { weeks of training: } \\
\mathrm{VO}_{2 \text { peak }} \\
\mathrm{O}_{2} \text { pulse } \\
\text { GXT duration } \\
\text { aerobic economy } \\
\text { after } 10 \text { weeks of training: } \\
\text { anaerobic threshold }\end{array}$ & $\begin{array}{l}+21 \% \\
\text { increase } \\
\text { increase } \\
\text { increase } \\
\text { increase }\end{array}$ & $\begin{array}{l}\leq 0.025^{\mathrm{b}} \\
\leq 0.025^{\mathrm{b}} \\
\leq 0.025^{\mathrm{b}} \\
\leq 0.001^{\mathrm{b}} \\
\leq 0.05^{\mathrm{b}}\end{array}$ \\
\hline $\begin{array}{l}\text { Hagberg et al. } \\
{[17](1983)} \\
\text { USA }\end{array}$ & 12 & $\begin{array}{l}\text { exercise }(n=6) \\
\text { control }(n=6)\end{array}$ & NDT & AER & $\begin{array}{l}\text { calisthenics, cycle } \\
\text { erg., walking } \\
\leq 30 \mathrm{~min} \\
50-85 \% \mathrm{VO}_{2 \text { peak }} \\
3-5 \times / \text { week }\end{array}$ & $\begin{array}{l}14 \pm 5 \\
\text { months }\end{array}$ & $\begin{array}{l}\mathrm{VO}_{2 \text { peak }} \\
\text { GXT duration } \\
\text { hemoglobin concentration } \\
\text { hematocrit concentration } \\
\text { SBP } \\
\text { DBP in }(\mathrm{n}=3) \text { patients } \\
\quad \text { with diastolic HT }\end{array}$ & $\begin{array}{l}+17 \% \\
+44 \% \\
\text { increase } \\
\text { increase } \\
\text { decrease } \\
\text { decrease }\end{array}$ & $\begin{array}{l}\leq 0.05^{\mathrm{b}} \\
\leq 0.01^{\mathrm{b}} \\
\leq 0.01^{\mathrm{b}} \\
\leq 0.01^{\mathrm{b}} \\
\leq 0.01^{\mathrm{b}} \\
\leq 0.05^{\mathrm{b}}\end{array}$ \\
\hline $\begin{array}{l}\text { Carney et al. } \\
\text { [18] (1983) } \\
\text { USA }\end{array}$ & 8 & $\begin{array}{l}\text { exercise }(n=4) \\
\text { control }(n=4)\end{array}$ & NDT & AER & $\begin{array}{l}\text { cycle ergometer, } \\
\text { walking, jogging } \\
50-60 \% \mathrm{VO}_{2 \text { peak }}, \\
3 \times / \text { week }\end{array}$ & 6 months & $\begin{array}{l}\mathrm{VO}_{2 \text { peak }} \\
\mathrm{GXT} \text { duration } \\
\text { depression/anxiety/hostility } \\
\text { frequency and enjoyment of } \\
\quad \text { pleasant activities }\end{array}$ & $\begin{array}{l}\text { no } \Delta \\
+28 \% \\
\text { decrease } \\
\text { Increase }\end{array}$ & $\begin{array}{l}- \\
<0.06^{\mathrm{b}} \\
<0.06^{\mathrm{b}} \\
<0.06^{\mathrm{b}}\end{array}$ \\
\hline $\begin{array}{l}\text { Painter et al. } \\
\text { [19] (1986) } \\
\text { USA }\end{array}$ & 20 & $\begin{array}{l}\text { exercise }(n=14) \\
\text { control }(n=6)\end{array}$ & ID & AER & $\begin{array}{l}\text { cycle ergometer } \\
65-85 \% \text { of } \mathrm{VO}_{2 \text { peak }} \\
30-45 \mathrm{~min}, 3 \times / \text { week }\end{array}$ & 6 months & $\begin{array}{l}\text { after } 3 \text { months of training: } \\
\mathrm{VO}_{2 \text { peak }} \\
\text { after } 6 \text { months of training: } \\
\mathrm{VO}_{2 \text { peak }} \\
\text { vertical work capacity }\end{array}$ & $\begin{array}{l}+17 \% \\
+23 \% \\
+40 \%\end{array}$ & $\begin{array}{l}\leq 0.05^{\mathrm{b}} \\
\leq 0.05^{\mathrm{b}} \\
\leq 0.05^{\mathrm{b}}\end{array}$ \\
\hline $\begin{array}{l}\text { Painter et al. } \\
\text { [20] (2000) } \\
\text { Painter et al. } \\
\text { [21] (2000) } \\
\text { USA }\end{array}$ & 286 & $\begin{array}{l}\text { exercise } \\
\text { control }\end{array}$ & HB\&ID & СОМВО & $\begin{array}{l}\text { HB: walking/cycle } \\
\text { ergometer to tolerance } \\
\text { and strength training } \\
5-6 \times / \text { weeks, } 8 \text { weeks } \\
\text { ID }(8 \text { weeks): } \\
\text { cycle erg to tolerance } \\
\leq 30 \mathrm{~min}, 3 \times / \text { week, } \\
8 \text { weeks }\end{array}$ & 16 weeks & $\begin{array}{l}\text { habitual gait speed } \\
\text { fastest gait speed } \\
\text { sit-to-stand speed } \\
\text { 6-min walk distance } \\
\text { SF-36 - physical functioning } \\
\text { SF-36 - role physical } \\
\text { SF-36 - general health } \\
\text { SF-36 - bodily pain } \\
\text { SF-36 - physical component }\end{array}$ & $\begin{array}{l}\text { increase } \\
\text { increase } \\
\text { increase } \\
\text { increase } \\
\text { increase } \\
\text { increase } \\
\text { increase } \\
\text { increase } \\
\text { increase }\end{array}$ & $\begin{array}{l}\leq 0.021^{\mathrm{a}} \\
\leq 0.001^{\mathrm{a}} \\
\leq 0.05^{\mathrm{a}} \\
\leq 0.05^{\mathrm{a}} \\
\leq 0.004^{\mathrm{a}} \\
\leq 0.001^{\mathrm{a}} \\
\leq 0.05^{\mathrm{a}} \\
\leq 0.003^{\mathrm{a}} \\
\leq 0.001^{\mathrm{a}}\end{array}$ \\
\hline $\begin{array}{l}\text { Miller et al. } \\
\text { [22] (2002) } \\
\text { USA }\end{array}$ & 75 & $\begin{array}{l}\text { exercise }(n=40) \\
\text { control }(n=35)\end{array}$ & ID & AER & $\begin{array}{l}\text { cycle ergometer } \\
\text { to tolerance } \\
\leq 30 \mathrm{~min}, 3 \times / \text { week }\end{array}$ & 6 months & $\begin{array}{l}\text { blood pressure } \\
\mathrm{n} \text { antihypertensive } \\
\quad \text { medications } \\
\text { expenditure on anti-HT } \\
\quad \text { medications }\end{array}$ & $\begin{array}{l}\text { no } \Delta \\
-36 \% \\
\text { decrease }\end{array}$ & $\begin{array}{l}- \\
0.018^{\mathrm{a}} \\
0.005^{\mathrm{a}}\end{array}$ \\
\hline $\begin{array}{l}\text { Moug et al. } \\
{[23](2004)} \\
\text { Scotland }\end{array}$ & 17 & $\begin{array}{l}\text { exercise }(n=10) \\
\text { control }(n=7)\end{array}$ & ID & AER & $\begin{array}{l}\text { cycle ergometer } \\
60-85 \% \mathrm{VO}_{2 \text { peak }} \\
45-60 \mathrm{~min}, 2 \times / \text { week }\end{array}$ & 6 weeks & $\begin{array}{l}\text { leg extension strength } \\
\text { depression } \\
\text { anxiety }\end{array}$ & $\begin{array}{l}\text { no } \Delta \\
\text { no } \Delta \\
\text { decrease }\end{array}$ & $\begin{array}{l}- \\
- \\
<0.05^{\mathrm{b}}\end{array}$ \\
\hline
\end{tabular}

NDT = Nondialysis time; ID = intradialytic; $\mathrm{HB}=$ home based; $\mathrm{AER}$ = aerobic training; $\mathrm{COMBO}$ = aerobic plus lower-intensity strength training; $\mathrm{AT}$ = anerobic threshold; GXT = graded exercise test $\mathrm{SBP}=$ systolic blood pressure; $\mathrm{DBP}=$ diastolic blood pressure .

${ }^{a}$ Significant over time vs. control group.

${ }^{\mathrm{b}}$ Significant vs. baseline value.

\section{Modality}

Nineteen trials $(19 / 29,66 \%)$ involved aerobic training as the sole exercise modality $[5-8,11,15-19,22-30$, 34, 37, 38]. Cycle ergometer training, walking/jogging, aerobics, calisthenics, swimming, and ball games were reported among these trials. Nine trials $(9 / 29,31 \%)$ com- bined aerobic training with some form of strength training $[9,10,12,20,21,31-33,35,36]$. Strength training interventions in these trials were generally not adequately described $[9,31,32,36]$, were of low intensity $[9,10$, $12,20,21,31-33,35,36]$, and/or involved lower extremity training only $[12,33,35]$. Only one trial to date $[15$, 
Table 3. Randomized controlled trials of exercise training in hemodialysis

\begin{tabular}{|c|c|c|c|c|c|c|c|c|c|}
\hline \multirow{2}{*}{$\begin{array}{l}\text { Authors (year) } \\
\text { country }\end{array}$} & \multirow[t]{2}{*}{$\mathrm{n}$} & \multirow[t]{2}{*}{ Study groups (n) } & \multicolumn{4}{|c|}{ Exercise intervention } & \multicolumn{3}{|l|}{ Outcomes } \\
\hline & & & delivery & modality & prescription & duration & variable & $\%$ change & $\mathrm{p}$ value \\
\hline $\begin{array}{l}\text { Goldberg et al. } \\
\text { [24] (1983) } \\
\text { Goldberg et al. } \\
\text { [25] (1986) } \\
\text { Harter et al. } \\
\text { [26] }(1985) \\
\text { USA }\end{array}$ & 25 & $\begin{array}{l}\text { exercise }(n=14) \\
\text { control }(n=11)\end{array}$ & NDT & AER & $\begin{array}{l}\text { walking, cycle erg. } \\
50-80 \% \mathrm{VO}_{2 \text { peak }} \\
45-60 \mathrm{~min}, 3 \times / \text { week }\end{array}$ & 12 months & $\begin{array}{l}\mathrm{VO}_{2 \text { peak }} \\
\mathrm{GXT} \text { duration } \\
\text { plasma triglyceride } \\
\text { VLDL triglyceride } \\
\text { VLDL cholesterol } \\
\text { HDL } \\
\text { glucose disappearance rate } \\
\text { insulin affinity } \\
\text { hematocrit } \\
\text { RBC mass } \\
\text { hemoglobin } \\
\text { basal insulin levels } \\
\text { RBC survival } \\
\text { Beck depression inventory }\end{array}$ & $\begin{array}{l}+17-21 \% \\
+26 \% \\
-29-33 \% \\
-30-38 \% \\
-16 \% \\
+16-20 \% \\
+35-42 \% \\
+25-70 \% \\
+27 \% \\
+27 \% \\
+16-20 \% \\
-21 \% \\
+46 \% \\
-42 \%\end{array}$ & $\begin{array}{l}\leq 0.01^{\mathrm{b}} \\
\leq 0.01^{\mathrm{b}} \\
\leq 0.01^{\mathrm{b}} \\
\leq 0.05^{\mathrm{b}} \\
\leq 0.02^{\mathrm{b}} \\
\leq 0.05^{\mathrm{b}} \\
\leq 0.02^{\mathrm{b}} \\
\leq 0.01^{\mathrm{b}} \\
\leq 0.02^{\mathrm{b}} \\
\leq 0.01^{\mathrm{b}} \\
\leq 0.01^{\mathrm{b}} \\
\leq 0.05^{\mathrm{b}} \\
\leq 0.02^{\mathrm{b}} \\
\leq 0.01^{\mathrm{b}}\end{array}$ \\
\hline $\begin{array}{l}\text { Carney et al. } \\
\text { [27] (1987) } \\
\text { USA }\end{array}$ & 21 & $\begin{array}{l}\text { exercise }(n=11) \\
\text { social support }(n=10)\end{array}$ & $\begin{array}{l}\text { NDT\& } \\
\text { HB }\end{array}$ & AER & $\begin{array}{l}\text { calisthenics, cycle } \\
\text { erg., walking } \\
60-80 \% \mathrm{VO}_{2 \text { peak }} \\
45-60 \mathrm{~min}, 3 \times / \text { week }\end{array}$ & 6 months & $\begin{array}{l}\text { Beck depression inventory } \\
\text { prevalence of clin. } \\
\text { depression } \\
\mathrm{VO}_{2 \text { peak }}\end{array}$ & $\begin{array}{l}\text { decrease } \\
\text { decrease } \\
+20 \%\end{array}$ & $\begin{array}{l}\leq 0.05^{\mathrm{a}} \\
\leq 0.05^{\mathrm{a}} \\
\mathrm{NR}\end{array}$ \\
\hline $\begin{array}{l}\text { Akiba et al. } \\
\text { [28] (1995) } \\
\text { Japan }\end{array}$ & 20 & $\begin{array}{l}\text { exercise }(n=10) \\
\text { control }(n=10)\end{array}$ & NDT & AER & $\begin{array}{l}\text { cycle ergometer, } \\
\text { RPE } \geq 12 \\
10-20 \mathrm{~min}, 3 \times / \text { week }\end{array}$ & 12 weeks & $\begin{array}{l}\text { exercise group: } \\
\mathrm{VO}_{2 \text { peak }} \\
\mathrm{VO}_{2 \mathrm{AT}} \\
\text { lactate at } \mathrm{VO}_{2 \text { peak }} \\
\text { control group: } \\
\mathrm{VO}_{2 \text { max }} \\
\mathrm{VO}_{2 \mathrm{AT}}\end{array}$ & $\begin{array}{l}\text { no } \Delta \\
\text { no } \Delta \\
\text { increase } \\
\text { decrease } \\
\text { decrease }\end{array}$ & $\begin{aligned} & - \\
& - \\
< & 0.001^{\mathrm{b}} \\
< & 0.05^{\mathrm{b}} \\
< & 0.05^{\mathrm{b}}\end{aligned}$ \\
\hline $\begin{array}{l}\text { Kouidi et al. } \\
\text { [29] (1997) } \\
\text { Greece }\end{array}$ & 31 & $\begin{array}{l}\text { exercise }(n=20) \\
\text { control }(n=11)\end{array}$ & NDT & AER & $\begin{array}{l}\text { cycle ergometer, } \\
\text { walking/JOG, } \\
\text { calisthenics, aerobics, } \\
\text { swimming or ball } \\
\text { games } \\
50-70 \% \mathrm{VO}_{2 \text { peak, }} \\
90 \mathrm{~min}, 3-4 \times / \text { week }\end{array}$ & 6 months & $\begin{array}{l}\mathrm{VO}_{2 \text { peak }} \\
\text { GXT duration } \\
\text { Beck depression inventory } \\
\text { QLI - patient activity } \\
\text { QLI - daily living } \\
\text { QLI - health } \\
\text { QLI - support } \\
\text { QLI - outlook }\end{array}$ & $\begin{array}{l}\text { increase } \\
\text { increase } \\
\text { increase } \\
\text { increase } \\
\text { increase } \\
\text { increase } \\
\text { increase } \\
\text { increase }\end{array}$ & $\begin{array}{l}\leq 0.05^{\mathrm{b}} \\
\leq 0.05^{\mathrm{b}} \\
\leq 0.05^{\mathrm{a}} \\
\leq 0.05^{\mathrm{a}} \\
\leq 0.05^{\mathrm{a}} \\
\leq 0.05^{\mathrm{a}} \\
\leq 0.05^{\mathrm{a}} \\
\leq 0.05^{\mathrm{a}}\end{array}$ \\
\hline $\begin{array}{l}\text { Frey et al. } \\
\text { [30] (1999) } \\
\text { USA }\end{array}$ & 11 & $\begin{array}{l}\text { exercise }(n=5) \\
\text { control }(n=6)\end{array}$ & ID & AER & $\begin{array}{l}\text { cycle ergometer } \\
\leq 45 \mathrm{~min} \\
60-80 \% \mathrm{HR}_{\max } \\
3 \times / \text { week }\end{array}$ & 7 weeks & $\begin{array}{l}\mathrm{Kt} / \mathrm{V} \\
\text { kilocalorie intake } \\
\text { protein intake } \\
\text { prealbumin } \\
\text { transferrin }\end{array}$ & $\begin{array}{l}\text { no } \Delta \\
\text { no } \Delta \\
\text { no } \Delta \\
\text { no } \Delta \\
\text { no } \Delta\end{array}$ & $\begin{array}{l}- \\
- \\
- \\
-\end{array}$ \\
\hline $\begin{array}{l}\text { Deligiannis et al. } \\
\text { [31] (1999) } \\
\text { Greece }\end{array}$ & 60 & $\begin{array}{l}\text { exercise }(n=30) \\
\text { control }(n=30)\end{array}$ & NDT & COMBO & $\begin{array}{l}\text { calisthenics, aerobics, } \\
\text { swimming or ball } \\
\text { games, and strength } \\
\text { exercise } \\
50-70 \% \mathrm{VO}_{2 \text { peak }} \\
90 \mathrm{~min}, 3-4 \times / \text { week }\end{array}$ & 6 months & $\begin{array}{l}\mathrm{VO}_{2 \text { peak }} \\
\mathrm{GXT} \text { duration } \\
\mathrm{HRV} \text { index } \\
\mathrm{SD} \text { of R-R interval } \\
\mathrm{R}-\mathrm{R} \text { interval length } \\
\mathrm{n} \text { patients. with HRV } \\
\text { index }<25 \\
\text { n patients with } \\
\text { arrhythmias } \\
\quad \text { (Lown class }>\mathrm{II} \text { ) }\end{array}$ & $\begin{array}{l}+41 \% \\
+33 \% \\
+31 \% \\
+18 \% \\
+18 \% \\
-40 \% \\
\end{array}$ & $\begin{array}{l}<0.05^{\mathrm{b}} \\
<0.05^{\mathrm{b}} \\
<0.05^{\mathrm{b}} \\
<0.05^{\mathrm{b}} \\
<0.05^{\mathrm{b}} \\
<0.05^{\mathrm{a}}\end{array}$ \\
\hline \multirow[t]{2}{*}{$\begin{array}{l}\text { Deligiannis et al. } \\
\text { [32] (1999) } \\
\text { Greece }\end{array}$} & 38 & exercise $1(n=16)$ & NDT & COMBO & $\begin{array}{l}\text { calisthenics, steps, up } \\
\text { to } 70 \mathrm{~min} \text {, and strength } \\
\text { exercise, } 3 \times / \text { week }\end{array}$ & 6 months & $\begin{array}{l}\text { exercise } 1 \\
\text { left ventricle mass index } \\
\text { ejection fraction } \\
\text { stroke volume index } \\
\text { cardiac output index }\end{array}$ & $\begin{array}{l}+11 \% \\
+12 \% \\
+23 \% \\
+20 \%\end{array}$ & $\begin{array}{l}\leq 0.05^{\mathrm{a}} \\
\leq 0.01^{\mathrm{a}} \\
\leq 0.05^{\mathrm{a}} \\
\leq 0.05^{\mathrm{a}}\end{array}$ \\
\hline & & $\begin{array}{l}\text { exercise } 2(n=10) \\
\text { control }(n=12)\end{array}$ & НB & AER & $\begin{array}{l}\text { cycle ergometer } \\
30 \text { min at } 50-60 \% \\
\mathrm{HR}_{\max },>5 \times / \text { week }\end{array}$ & & $\begin{array}{l}\text { exercise } 2 \\
\text { no significant adaptations } \\
\text { vs. control group }\end{array}$ & & \\
\hline $\begin{array}{l}\text { DePaul et al. } \\
\text { [33] (2002) } \\
\text { Canada }\end{array}$ & 37 & $\begin{array}{l}\text { exercise }(n=20) \\
\text { placebo }(n=17)\end{array}$ & ID & COMBO & $\begin{array}{l}\text { cycle erg } \\
\text { Borg } \mathrm{RPE}=13 / 20 \\
<80 \mathrm{HR}_{\max }, 20 \mathrm{~min}, \& \\
\text { knee extensions (pre-dial } \\
3 \text { sets } \times 10 \text { reps at } \\
50 \% \text { of } 5 \mathrm{RM}, 3 \times / \text { week }\end{array}$ & 12 weeks & $\begin{array}{l}\text { submaximal ex. capacity } \\
\text { knee extension strength }\end{array}$ & $\begin{array}{l}\text { increase } \\
\text { increase }\end{array}$ & $\begin{array}{l}<0.02^{\mathrm{a}} \\
<0.02^{\mathrm{a}}\end{array}$ \\
\hline
\end{tabular}


Table 3 (continued)

\begin{tabular}{|c|c|c|c|c|c|c|c|c|c|}
\hline \multirow{2}{*}{$\begin{array}{l}\text { Authors (year) } \\
\text { country }\end{array}$} & \multirow[t]{2}{*}{$\mathrm{n}$} & \multirow[t]{2}{*}{ Study groups (n) } & \multicolumn{4}{|c|}{ Exercise intervention } & \multicolumn{3}{|l|}{ Outcomes } \\
\hline & & & delivery & modality & prescription & duration & variable & $\%$ change & $\mathrm{p}$ value \\
\hline \multirow{2}{*}{$\begin{array}{l}\text { Painter et al. } \\
\text { [34] (2002) } \\
\text { USA }\end{array}$} & \multirow[t]{2}{*}{48} & exercise+EPO $(n=12)$ & ID & AER & $\begin{array}{l}\text { cycle ergometer } \\
\text { RPE 12-14 }\end{array}$ & 5 months & $\begin{array}{l}\text { in post-HOC analysis } \\
\text { exercise groups: }\end{array}$ & & \\
\hline & & $\begin{array}{l}\text { exercise only }(\mathrm{n}=10) \\
\text { EPO only }(\mathrm{n}=12) \\
\text { control }(\mathrm{n}=14)\end{array}$ & ID & AER & $\begin{array}{l}30 \mathrm{~min}, 3 \times / \text { week } \\
\text { cycle ergometer } \\
\text { RPE } 12-14 \\
30 \mathrm{~min}, 3 \times / \text { week }\end{array}$ & & $\begin{array}{l}\mathrm{VO}_{2 \text { peak }} \\
\mathrm{SF}-36 \text {-physical } \\
\text { functioning }\end{array}$ & $\begin{array}{l}\text { increase } \\
\text { increase }\end{array}$ & $\begin{array}{l}\leq 0.028^{\mathrm{a}} \\
\leq 0.015^{\mathrm{a}}\end{array}$ \\
\hline \multirow{8}{*}{$\begin{array}{l}\text { Konstantinidou } \\
{[35](2002)} \\
\text { Greece }\end{array}$} & \multirow[t]{8}{*}{48} & exercise $1(n=16)$ & ND & СОМBO & $\begin{array}{l}\text { aerobic training } \\
50-70 \% \mathrm{VO}_{2 \text { peak }}\end{array}$ & 6 months & $\begin{array}{l}\text { exercise group } 1 \\
\mathrm{VO}_{2 \text { peak }}\end{array}$ & $+43 \%$ & $\leq 0.05^{\mathrm{a}}$ \\
\hline & & & & & $60 \mathrm{~min}$ and strength & & GXT duration & $+33 \%$ & $\leq 0.05^{\mathrm{a}}$ \\
\hline & & & & & exercise, $3 \times /$ week & & $\begin{array}{l}\mathrm{VE}_{\text {peak }} \\
\mathrm{VO}_{2 \mathrm{AT}}\end{array}$ & $\begin{array}{l}+41 \% \\
+37 \%\end{array}$ & $\begin{array}{l}\leq 0.05^{\mathrm{a}} \\
\leq 0.05^{\mathrm{a}}\end{array}$ \\
\hline & & exercise $2(n=10)$ & ID & COMBO & cycle ergometer & & exercise group 2 & & \\
\hline & & & & & Borg RPE = 13 & & $\mathrm{VO}_{2 \text { peak }}$ & & \\
\hline & & & & & 60-90 min, and lower & & GXT duration & $+22 \%$ & $\leq 0.05^{\mathrm{c}}$ \\
\hline & & & & & $\begin{array}{l}\text { body strength exercise } \\
3 \times / \text { week }\end{array}$ & & $\begin{array}{l}\mathrm{VE}_{\text {peak }} \\
\mathrm{VO}_{2 \mathrm{AT}}\end{array}$ & $\begin{array}{l}+12 \% \\
+18 \%\end{array}$ & $\begin{array}{l}\leq 0.05^{\circ} \\
\leq 0.05^{\mathrm{c}}\end{array}$ \\
\hline & & $\begin{array}{l}\text { exercise } 3(n=10) \\
\text { control }(n=12)\end{array}$ & HB & AER & $\begin{array}{l}\text { cycle erg., } 50-60 \% \\
\text { HR }_{\max } \text {, } \\
30 \text { min, } 5 \times / \text { week }\end{array}$ & & $\begin{array}{l}\text { exercise group } 3 \\
\mathrm{VO}_{2 \text { peak }} \\
\mathrm{GXT} \text { duration } \\
\mathrm{VE}_{\text {peak }} \\
\mathrm{VO}_{2 \mathrm{AT}}\end{array}$ & $\begin{array}{l}+17 \% \\
+14 \% \\
\text { increase } \\
+8 \%\end{array}$ & $\begin{array}{l}\leq 0.05^{\mathrm{c}} \\
\leq 0.05^{\mathrm{c}} \\
\leq 0.05^{\mathrm{c}} \\
\leq 0.05\end{array}$ \\
\hline \multirow{12}{*}{$\begin{array}{l}\text { Kouidi et al. } \\
\text { [36] (2004) } \\
\text { Greece }\end{array}$} & \multirow[t]{12}{*}{34} & exercise $1(n=16)$ & ND & СОМBO & $\begin{array}{l}\text { aerobic training } \\
50-70 \% \mathrm{VO}_{2 \text { peak }} \text {, }\end{array}$ & 4 years & $\begin{array}{l}\text { after } 1 \text { year } \\
\text { exercise } 1 \text { /exercise } 2\end{array}$ & & \\
\hline & & & & & $60 \mathrm{~min}$, and strength & & $\mathrm{VO}_{2 \text { peak }}$ & $+47 \% /+34 \%$ & $\leq 0.05^{\mathrm{d}}$ \\
\hline & & & & & training, $3 \times /$ week & & GXT duration & $+38 \% /+26 \%$ & $\leq 0.05^{\mathrm{d}}$ \\
\hline & & & & & & & $V E_{\text {peak }}$ & $+24 \% /+13 \%$ & $\leq 0.05^{\mathrm{a}}$ \\
\hline & & & & & & & $\mathrm{VO}_{2 \mathrm{AT}}$ & $\begin{array}{l}+39 \% /+29 \% \\
\text { increase }\end{array}$ & $\leq 0.05^{\mathrm{d}}$ \\
\hline & & & & & & & Weak & & \\
\hline & & exercise $2(n=18)$ & ID & СOMBO & cycle ergometer & & after 4 years & & \\
\hline & & & & & $60-90 \mathrm{~min}$, and lower & & $\mathrm{VO}_{2 \text { peak }}$ & $+70 \% /+50 \%$ & $\leq 0.05^{\mathrm{d}}$ \\
\hline & & & & & body strength exercise & & GXT duration & $+53 \% /+43 \%$ & $\leq 0.05^{\mathrm{d}}$ \\
\hline & & & & & $3 \times /$ week & & $V E_{\text {peak }}$ & $+43 \% /+26 \%$ & $\leq 0.05^{\mathrm{d}}$ \\
\hline & & & & & & & $\mathrm{VO}_{2 \mathrm{AT}}$ & $+52 \% /+42 \%$ & $\leq 0.05^{\mathrm{d}}$ \\
\hline & & & & & & & HKeak & morease & $\leq 0.05$ \\
\hline \multirow{4}{*}{$\begin{array}{l}\text { Molsted et al. } \\
\text { [37] (2004) } \\
\text { Denmark }\end{array}$} & \multirow[t]{4}{*}{33} & exercise $(n=22)$ & NDT & AER & $\begin{array}{l}\text { step exercises, cycling, } \\
\text { aerobics, RPE 14-17 }\end{array}$ & 5 months & $\begin{array}{l}\mathrm{VO}_{2 \text { peak }} \\
\text { SF-36 - physical }\end{array}$ & increase & $\leq 0.012^{\mathrm{a}}$ \\
\hline & & control $(\mathrm{n}=11)$ & & & $60 \mathrm{~min}, 2 \times /$ week & & functioning & increase & $\leq 0.01^{\mathrm{a}}$ \\
\hline & & & & & & & $\begin{array}{l}\text { SF-36 - bodily pain } \\
\text { SF-36 - physical }\end{array}$ & improved & $\leq 0.03^{\mathrm{a}}$ \\
\hline & & & & & & & component & increase & $\leq 0.004^{\mathrm{a}}$ \\
\hline \multirow{4}{*}{$\begin{array}{l}\text { Parsons et al. } \\
\text { [38] (2004) } \\
\text { Canada }\end{array}$} & \multirow[t]{4}{*}{13} & exercise $(n=6)$ & ID & AER & cycle ergometer & 8 weeks & exercise group & & \\
\hline & & & & & $40-50 \%$ maximum & & maximal work capacity & no $\Delta$ & - \\
\hline & & control $(\mathrm{n}=7)$ & & & $45 \mathrm{~min}, 3 \times /$ week & & blood urea clearance & no $\Delta$ & - \\
\hline & & & & & & & $\begin{array}{l}\text { dialysate urea clearance } \\
\text { QOL }\end{array}$ & $\begin{array}{l}\text { increase } \\
\text { no } \Delta\end{array}$ & $\begin{array}{l}\leq 0.05^{\circ} \\
-\end{array}$ \\
\hline
\end{tabular}

NDT = Nondialysis time; ID = intradialytic; $\mathrm{HB}$ = home based; $\mathrm{AER}=$ aerobic training; $\mathrm{COMBO}=$ aerobic plus lower-intensity strength training; $\mathrm{PRT}$ = progressive resistance training; $\mathrm{QLI}=$ Quality of Life Index; $\mathrm{HRV}=$ heart rate variability; $\mathrm{VE}_{\text {peak }}=$ peak ventilation; $\mathrm{HR}_{\text {peak }}=$ peak heart rate; $\mathrm{GXT}=$ graded exercise test.

${ }^{\text {a }}$ Significant change over time versus comparison group(s).

${ }^{\mathrm{b}}$ Significant vs. baseline values within group.

${ }^{\mathrm{c}}$ Significant vs. control group only.

${ }^{\mathrm{d}}$ Significant change over time between exercise group 1 and exercise group 2. 
16] has prescribed a progressive resistance training (PRT) intervention targeting the upper and lower extremities performed at a relatively high intensity (10-15 $\mathrm{RM}$ ) as currently recommended for improving the musculoskeletal fitness of healthy adults and the elderly [43].

Delivery

Exercise training has been prescribed:

- In non-dialysis time at a training center in $12 / 29$ trials (41\%) [5-7, 9, 15-18, 24-26, 28, 29, 31, 37].

- During HD treatment in $11 / 29$ trials $(38 \%)[8,10,11$, $12,19,22,23,30,33,34,38]$.

- In nondialysis time at a training center + at home in $2 / 29$ trials $(7 \%)[13,14,27]$.

- During HD treatment + at home in $1 / 32$ trial (3\%) [20, 21].

Additionally, 3 RCTs $(3 / 29,10 \%)$ assigned patients into treatment groups that trained in separate locations, including in non-dialysis time in a training center [32, 35, $36]$, at home [32, 35] and/or during $\operatorname{HD}[32,35,38]$.

\section{Frequency}

Exercise training was typically prescribed for 3-4 sessions/week (tables 1-3) [8, 9, 11, 16, 18, 19, 22, 24-36, $38]$. In two RCTs $(2 / 29,7 \%)$, subjects randomized to home-based training were prescribed $\geq 5$ training sessions/week [32, 35]. Painter et al. [20, 21] prescribed home-based exercise 5-6 sessions/week for 8 weeks followed by intradialytic training 3 sessions/week for 8 weeks. One trial $(1 / 29,3 \%)$ did not report on the frequency of exercise training $[5,6]$.

\section{Intensity}

Aerobic training interventions were generally of moderate intensity and progressed according to tolerance as the conditioning of the patient improved (tables 1-3). Two trials, however, prescribed and maintained aerobic training at a relatively low intensity $(\leq 60 \%$ of maximal effort) $[18,38]$. Additionally, several trials did not mention how aerobic training intensity was gauged $[9,10,11$, 22].

Strength training interventions were generally prescribed at a low to moderate intensity $[9,10,12,20,21$, $31-33,35,36]$, with 1 trial $(1 / 29,3 \%)$ prescribing higherintensity PRT $[15,16]$ (tables 1-3).

Duration of Aerobic Training Sessions

In general, the duration of aerobic training sessions ranged from 30 to $60 \mathrm{~min} / \mathrm{session}$, with a few trials ex- ceeding this duration with the inclusion of warm-up and cool-down periods $[32,35,36]$. Two trials $(2 / 29,7 \%)$ maintained aerobic training sessions to $\leq 20 \mathrm{~min} / \mathrm{session}$ $[28,33]$. One trial $(1 / 29,3 \%)$ did not define the duration of aerobic training per session $[5,6]$.

\section{Supervision}

Eighteen trials $(18 / 29,62 \%)$ reported that qualified health professionals including study personnel supervised exercise training sessions [5-10, 12-16, 24-26, $29,31-37]$. Nine trials did not provide details regarding supervision $[11,17,18,22,23,27,28,30,38]$. In one trial by Painter et al. [20, 21], which combined homebased and intradialytic training, subjects trained independently in both locations. In another trial by Painter et al. [19], the first half (3 months) of an intradialytic cycling program was directly supervised, while the latter half was not. Home-based interventions were never directly monitored however regular contact with study personnel was reported as being provided [13, 14, 20, $21,27,32,35]$.

\section{Compliance}

Nine trials $(9 / 29,31 \%)$ provided information regarding compliance to exercise training (e.g. sessions attended). In these trials [7, 8, 13-15, 19, 22, 23, 29, 37], compliance ranged from fair (43\%) [7] to excellent (99\%) [23]. However, no trial to date has provided an a priori definition of 'compliance' within their methods section.

\section{Adverse Events}

Thirteen trials $(13 / 29,45 \%)$ have reported that no serious complications have resulted from participation in the prescribed exercise intervention [7, 9, 10, 12-14, 22, $23,29,32,34-36]$. Trials have noted that hypotension has been induced by exercise training $[33,38]$ though not always presenting with regularity [13-15]. One trial reported an acute gastrointestinal hemorrhage in an exercising subject [16]. DePaul et al. [33] reported more adverse events in exercising versus nonexercising patients, citing complaints such as fatigue $(n=1)$, soreness $(n=1)$, hypotension $(\mathrm{n}=1)$, foot ulcer $(\mathrm{n}=1)$ and foot pain $(\mathrm{n}=$ 1). No other serious adverse events have been reported in the 29 trials presented in this review. However, to date, no trial has provided an a priori definition of 'adverse event' within their methods section. 


\section{Adaptations to Exercise Training in Hemodialysis}

Documented adaptations to exercise training in the 29 trials reviewed are presented in tables 1-3. A synopsis of these findings highlights some important physiological, functional, and psychological benefits of exercise training in this cohort.

\section{Physiological Adaptations to Exercise Training}

The Cardiorespiratory System and Aerobic Capacity

Several trials have reported that HD patients can significantly increase peak oxygen consumption $\left(\mathrm{VO}_{2 \text { peak }}\right)$ $17-23 \%$ by performing aerobic training during nondialysis time [16, 17, 24-27, 29], during dialysis [19], and at home [35]. By contrast, a few trials have reported no significant improvement of $\mathrm{VO}_{2 \text { peak }}$ with aerobic training, which may perhaps be due to prescribing low intensity $\left(<60 \% \mathrm{VO}_{2 \text { peak }}\right)$ [24] and/or short duration (10-20 min/ session) training [28]. Moreover, it has been well documented that oxygen consumption in this cohort is limited at the peripheral level (i.e. at the skeletal muscle) [40], which is not optimally enhanced with aerobic exercise training alone [8]. In support of this, the magnitude of improvement in $\mathrm{VO}_{2 \text { peak }}$ secondary to combined (aerobic and strength) training (41-48\%) $[9,31,32,35,36]$ is notably superior to studies prescribing aerobic training only (17-23\%). No studies have directly compared aerobic and strength training evaluating this outcome, or assessed $\mathrm{VO}_{2 \text { peak }}$ after isolated strength training. Central, intermediary and peripheral cardiorespiratory system adaptations to exercise training documented in the literature are presented in tables 1-3.

\section{Cardiac Functioning}

Deligiannis et al. [32] in a RCT demonstrated that 6 months of combined training on nondialysis days improved left ventricle mass index, ejection fraction, cardiac output index, and stroke volume index. Another RCT by the same authors [31] revealed that 6 months of combined training could significantly increase heart rate variability index and the standard deviation of the R-R interval, while reducing the prevalence of arrhythmias (Lown class $>$ II).

Muscle Architecture and Neuromuscular Control

Kouidi et al. [9] reported in an uncontrolled trial of 7 patients that cross-sectional area of type I and II muscle fibers obtained from the vastus lateralis significantly increased, 2,831 \pm 846 to $3,565 \pm 764 \mu \mathrm{m}^{2}$ and 2,683 \pm
763 to $3,319 \pm 1,049 \mu \mathrm{m}^{2}$, respectively, with 6 months of combined aerobic and strength training. Further, the ratio of type I to type II fibers improved from 54.6:45.4 to $31.6: 68.4$, which is reported to be near normal $(1: 2)$ for this biopsy site. Ultrastructural analysis revealed that the muscle appeared more normal, including positive adaptations of the capillaries and mitochondria. The authors also noted activation of satellite cells and an increased number of leukocytes and natural killer cells. Motor conduction of the peroneal nerve also significantly improved $(p<0.05)$. By contrast, Moore et al. [8] observed no hypertrophy secondary to 6 months of intradialytic aerobic training, which is not unexpected given that aerobic training is not the preferred exercise modality for eliciting myogenic adaptation.

\section{Components of Metabolic Syndrome}

Miller et al. [22] demonstrated that hypertensive patients could significantly reduce predialysis and postdialysis systolic blood pressure after 3 months of intradialytic cycling. The reduction in blood pressure was accompanied by a reduction in antihypertensive medications $(-36 \%, p<0.018)$ resulting in a cost savings of USD 885 per patient annually. Additional trials have observed reduced resting blood pressure [15, 17, 32], and blood pressure during maximal exercise [32] with $>3$ months of aerobic, or combined training. Other studies have expressed such findings anecdotally $[5,6,19,24]$.

Goldberg and colleagues [5, 6, 24-26] demonstrated that nondiabetic HD patients could significantly reduce fasting plasma glucose and insulin concentrations, while significantly increasing insulin-binding affinity and glucose disappearance rate with $8-12$ months of aerobic training. The authors [5, 6, 24-26] also reported increased fasting plasma high-density lipoprotein (HDL) cholesterol, reduced very-low-density lipoprotein (VLDL), reduced VLDL triglyceride, and reduced total plasma triglyceride secondary to aerobic exercise training.

To date there have been no studies investigating the effects of exercise training on visceral obesity in this cohort, or studies of insulin sensitivity and glucose control in diabetic patients receiving maintenance HD.

Dialysis Adequacy

One uncontrolled trial has demonstrated an improvement in dialysis adequacy $(\mathrm{Kt} / \mathrm{V})$ with 6 months of intradialytic aerobic training using cycle ergometers [11]. By contrast, two trials implementing shorter training durations ( 7 and 8 weeks) have not observed an improvement in $\mathrm{Kt} / \mathrm{V}[30,38]$. Evidence suggests that a single, acute 
bout of intradialytic cycling can significantly enhance the removal of urea, creatinine and potassium during HD by significantly reducing post-dialysis rebound of these damaging solutes [41]. Intradialytic exercise training could enhance dialysis adequacy chronically via this same mechanism [3]. However, this hypothesis has not yet been rigorously investigated within a longitudinal RCT [3].

\section{Functional Adaptations to Exercise Training Muscular Strength}

Headley et al. [13] and Nindl et al. [14] prescribed high-intensity PRT to elicit improvements in muscular strength in HD patients. Four additional trials [9, 10, 12, 33] reported improved muscular strength with regimens involving lower-intensity strength training. By contrast, Moug et al. [23] reported no significant improvement of lower body strength secondary to 6 weeks of intradialytic cycling. This finding is not unexpected given that aerobic training is not the preferred modality for improving muscular strength, unlike resistance training [42].

Functional Performance

Hemodialysis patients can significantly improve exercise capacity (i.e. 6-min walk distance) secondary to PRT [13] or combined training [10,21]. Other functional performance outcomes reported include increased maximal walking speed $[13,20,21]$, habitual walking speed [20, 21], and sit-to-stand movement speed [13, 20, 21].

\section{Disability}

Independence in activities of daily living has not specifically been measured after exercise in this cohort. However, in the longest trial of exercise training conducted with HD patients, Kouidi et al. [36] demonstrated that combined training on non-dialysis days significantly improved the likelihood of returning to work after 1 and 4 years of training.

\section{Psychological Adaptations to Exercise Training \\ Depression}

Carney et al. [27], in a RCT, showed that aerobic training alleviated depression to a greater extent than participation in a social support group in this cohort, as evaluated by the Beck Depression Inventory (BDI) [43] (p < $0.05)$. Significantly reduced BDI scores following 3-12 months of aerobic exercise training have been observed in other trials [24, 25, 29]. Kouidi et al. [29] suggested that the most severely depressed patients benefited to the greatest extent.

Exercise for Hemodialysis Patients
Quality of Life

Several trials of exercise training in HD patients have evaluated Medical Outcomes Trust Short-Form 36 (SF36) [44] scores as health-related QOL outcome measures. Improved perceptions of 'physical functioning' have been observed secondary to $3-5$ months of aerobic $[34,37]$ and combined training $[12,20,21]$. Painter et al $[20,21]$ reported improvements in other SF-36 QOL domains including: 'role physical' [20, 21], 'bodily pain' [20, 21], 'general health' [20, 21], 'vitality' [20], and the 'physical component scale' $[20,21]$, especially in patients with low baseline perceptions of physical functioning. Oh-Park et al. [12] showed improved 'mental health' scores with combined training performed during HD. One trial did not yield improvements in any SF-36 scores [33]. The authors [33] speculated that the lack of significance could be due to the fact that their samples had high functional status at baseline and/or their study was inadequately powered. Improved measures of QOL have been ascertained in this cohort using other scales [31], including the Spitzer QOL Index (QLI) [45].

\section{Discussion}

Overall, the evidence gathered in this critical review suggests that appropriately prescribed exercise involving aerobic and/or resistance training modalities, is safe and beneficial for HD patients. Planned exercise can induce a myriad of positive health and clinical adaptations in this cohort, which may be associated with enhanced quality and quantity of life. However, current limitations within this body of the literature may be partially responsible for the fact that exercise training is not routinely recommended or prescribed in this cohort by practitioners [3, 4]. Despite nearly 3 decades of research demonstrating the benefits of exercise in ESRD, advocacy for exercise has been notably absent from official position stands and policy documents until the publication of a brief supportive statement in the recent Kidney Disease Outcomes Quality Initiative (KDOQI) in April 2005 [46].

Thirteen trails $(13 / 29,45 \%)$ reviewed were RCTs. Several of these RCTs were methodologically limited according to current standards of reporting [47]. Limitations were evident with respect to: statistical analyses where only 2 studies mentioned utilization of intention-to-treat strategy; the limited involvement of blinded outcomes assessors; and the inadequate reporting of subject characteristics, interventions, and outcome measures, includ- 
ing safety and compliance. Further, the external validity of $9 / 13(69 \%)$ RCTs reviewed is compromised by the fact that these trials excluded diabetics. Diabetes has become the leading cause of ESRD in the United States affecting approximately $45 \%$ of newly diagnosed patients [1]. Currently, over $35 \%$ of patients with ESRD in the US are diagnosed diabetics [1].

Thorough and standardized reporting [47] is required of future clinical trials of exercise training in HD patients. Subject characteristics should be clearly described, including the etiology of renal failure and comorbidities. Interventions should be thoroughly defined with respect to frequency, intensity, modality, session duration, delivery, and supervision. This is essential for determining the exercise prescription required to positively affect specific outcomes. Clearly, the fact that some trials we reviewed observed no effect of exercise training on certain outcomes $[8,15,23,28,30,38]$ does not imply that exercise, in general, is ineffective in this cohort, but rather suggests that the exercise dose and/or modality prescribed was insufficient to positively affect the desired measure. Compliance to training should be defined a priori to determine the feasibility and generalizability of prescribing exercise training in this patient population. Thorough reporting of adverse events, including a priori definitions, is necessary to determine the risk to benefit ratio of exercise training in this cohort, which is suggested to be favorable among other chronically diseased populations [42].

The documented adaptations to exercise training in the 29 trials reviewed represent important areas of benefit to the HD population. The physiological, functional, and psychological adaptations induced by exercise may be associated with reduced cardiovascular risk profile, improved QOL, and extended lifespan. At present, however, robustly designed studies are required to further evaluate many of these, and other health-related and clinical outcomes, including skeletal muscle wasting, osteoporosis, the malnutrition-inflammatory complex, dialysis adequacy, metabolic syndrome, endothelial dysfunction, disability, depression, self-efficacy and QOL. Future investigations should also be conducted explicitly with targeted subpopulations within this cohort, including those suffering from clinical depression, obesity, hypertension, and insulin resistance/diabetes. For example, there are currently no trials evaluating insulin resistance/glucose homeostasis in diabetics on HD, nor are there trials of exercise in patients with clinically diagnosed depressive illness. Additionally, few studies have specifically targeted patients $>65$ years, an increasingly large cohort with a greater burden of complex comorbidities which may impact on both feasibility and benefit of exercise training interventions.

Trials prescribing aerobic and resistance training modalities, independently and in combination, should be conducted. It could be hypothesized that combined interventions elicit superior adaptations of $\mathrm{VO}_{2 \text { peak }}$ (central and peripheral) and other health-related outcomes than either intervention on its own; however, studies isolating each modality will be useful for determining which beneficial adaptations can be assigned to each modality specifically. At present, there is only one report involving PRT in this cohort $[13,14]$. This is a significant gap in the literature given the risk and critically important outcomes associated with skeletal muscle wasting in patients with chronic uremia [48]. PRT may also be a more feasible exercise modality in this cohort as patients with congestive and ischemic heart disease, who cannot engage in vigorous aerobic training, may be able to perform robust PRT safely. PRT is currently widely advocated and prescribed for health benefits in various healthy, and chronically diseased cohorts [42], though not in HD patients as yet.

Various methods of exercise delivery should continue to be investigated, compared and contrasted, as recently performed by Kouidi, Konstantinidou et al. [35, 36]. It should be noted, however, that training volume in these trials was not equated. Thus, the greater cardiorespiratory benefits achieved by patients training on nondialysis days could primarily be attributed to the fact that they received a greater volume of training. Future trials should therefore equate the volume of training to determine which method of training is more feasible, and beneficial. Novel exercise equipment customized to the HD setting will likely have to be developed to investigate such hypotheses.

Long-term behavioral change is the challenge to exercise prescription in most clinical cohorts, and patients receiving maintenance $\mathrm{HD}$ for the management of ESRD are no exception. Only 4 trials were identified which were $>6$ months in duration $[5,6,17,24-26,36]$, compliance was often not reported, and virtually no information was presented on psychological, demographic, or clinical predictors of adoption and adherence in the patients studied. Future studies can contribute to the successful dissemination of their research findings and overcome barriers to behavioral change if such analyses are conducted and compliance, as well as reasons for noncompliance, is carefully documented. 
The available literature supports the clinical utility of exercise participation for HD patients. Although methodological shortcomings exist, and gaps in knowledge are clearly evident in some specific areas, there is sufficient empirical published evidence to support the addition of exercise recommendations to clinical guidelines, as recently published by the KDOQI [46]. Further research is required to advance these guidelines toward the development of position stands on exercise prescription. There is no other available medical treatment with the capacity to induce beneficial adaptations across as wide a range of physiological, functional, psychological, and clinical domains as appears possible with sufficient doses of aerobic and resistance training. In addition, it appears possible to creatively modify the sedentary, often negative, and depressing ambiance of the typical HD unit by bringing the exercise treatment directly into this medical setting. Such complete integration of exercise and medicine is critical for its acceptance by practitioners as part of mainstream medical care, for enhancing compliance and safety, and perhaps for the actual improvement of dialysis adequacy itself.

\section{References}

1 United States Renal Data System (USRDS) 2004 Annual Data Report: Atlas of End-Stage Renal Disease in the United States. Bethesda, National Institutes of Health, National Institute of Diabetes and Digestive and Kidney Diseases, 2004.

2 The Scottish Renal Registry Links: Available at: http://www.show.scot.nhs.uk/srr/Links/ Registries.htm.

3 Cheema B, Smith B, Fiatarone Singh M: A rationale for intradialytic exercise training as standard clinical practice in ESRD. Am J Kidney Dis 2005;45:912-916.

-4 Johansen KL, Sakkas GK, Doyle J, Shubert T, Dudley RA: Exercise counselling practices among nephrologists caring for patients on dialysis. Am J Kidney Dis 2003;41:171-178.

$\checkmark 5$ Goldberg AP, Hagberg JM, Delmez JA, Florman RW, Harter HR: Effects of exercise training on coronary risk factors in hemodialysis patients. Proc Dial Transplant Forum 1979;9: 39-43.

-6 Goldberg AP, Hagberg JM, Delmez JA, Haynes ME, Harter HR: Metabolic effects of exercise training in hemodialysis patients. Kidney Int 1980;18:754-761.

-7 Shalom R, Blumenthal JA, Williams RS, McMurray RG, Dennis VW: Feasibility and benefits of exercise training in patients on maintenance hemodialysis. Kidney Int 1984;25: 958-963.

8 Moore GE, Parsons DB, Stray-Gundersen J, Painter PL, Brinker KR, Mitchell JH: Uremic myopathy limits aerobic capacity in hemodialysis patients. Am J Kidney Dis 1993;22:277287.

$\checkmark 9$ Kouidi E, Albani M, Natsis K, et al: The effects of exercise training on muscle atrophy in hemodialysis patients. Nephrol Dial Transplant 1998; 13:685-699.

$\checkmark 10$ Ridley J, Hoey K, Ballagh-Howes N: The exercise-during-hemodialysis program: Report on a pilot study. CANNT J 1999;9:20-26.
11 Zaluska A, Zaluska W, Bednarek-Skublewska A, Ksiazek A: Nutrition and hydration status improve with exercise training using stationary cycling during hemodialysis (HD) in patients with end-stage renal disease (ESRD). Ann Univ Mariae Curie Sklodowska [Med] 2002; 57:342-346.

12 Oh-Park M, Fast A, Gopal S, et al: Exercise for the dialyzed: aerobic and strength training during hemodialysis. Am J Phys Med Rehabil 2002;81:814-821.

13 Headley S, Germain M, Mailloux P, et al: Resistance training improves strength and functional measures in patients with end-stage renal disease. Am J Kidney Dis 2002;40: 355-364.

14 Nindl BC, Headley SA, Tuckow AP, et al: IGFI system responses during 12 weeks of resistance training in end-stage renal disease patients. Growth Horm IGF Res 2004; 14: 245-250.

15 Mustata S, Chan C, Lai L, Miller J: Impact of an exercise program on arterial stiffness and insulin resistance in hemodialysis patients. $\mathrm{J}$ Am Soc Nephrol 2004;15:2713-2718.

16 Zabetakis PM, Gleim GW, Saraniti A, Nicholas JA, Michelis MF: Long-duration submaximal exercise conditioning in hemodialysis patients. Clin Nephrol 1982;18:17-22.

17 Hagberg JM, Goldberg AP, Ehsani AA, Heath GW, Delmez JA, Harter HR: Exercise training improves hypertension in hemodialysis patients. Am J Nephrol 1983:3:209-212.

18 Carney RM, McKevitt PM, Goldberg AP, Hagberg J, Delmez JA, Harter HR: Psychological effects of exercise training in hemodialysis patients. Nephron 1983;33:179-181.

19 Painter PL, Nelson-Worel JN, Hill MM, et al: Effects of exercise training during hemodialysis. Nephron 1986;43:87-92.

20 Painter P, Carlson L, Carey S, Paul S, Myll J: Low-functioning hemodialysis patients improve with exercise training. Am J Kidney Dis 2000;36:600-608.
21 Painter P, Carlson L, Carey S, Paul SM, Myll $\mathrm{J}$ : Physical functioning and health-related quality-of-life changes with exercise training in hemodialysis patients. Am J Kidney Dis 2000; 35:482-492.

22 Miller B, Cress C, Johnson M, Nichols D, Schnitzler M: Exercise during hemodialysis decreases the use of antihypertensive medications. Am J Kidney Dis 2002;39:828-833.

23 Moug S, Grant S, Creed G, Boulton Jones M: Exercise during haemodialysis: West of Scotland pilot study. Scott Med J 2004;49:14-17.

24 Goldberg AP, Geltman EM, Hagberg JM, et al: Therapeutic benefits of exercise training for hemodialysis patients. Kidney Int 1983;24: S303-S309.

25 Goldberg AP, Geltman EM, Gavin JR, et al: Exercise training reduces coronary risk factors and effectively rehabilitates hemodialysis patients. Nephron 1986:42:311-316.

-26 Harter HR, Goldberg AP: Endurance exercise training: an effective therapeutic modality for hemodialysis patients. Med Clin N Am 1985; 69:159-175.

27 Carney R, Templeton B, Hong B, et al: Exercise training reduces depression and increases the performance of pleasant activities in hemodialysis patients. Nephron 1987;47:194-198.

-28 Akiba T, Matsui N, Shinohara S, Fujiwara H, Nomura T, Marumo F: Effects of recombinant human erythropoietin and exercise training on exercise capacity in hemodialysis patients. Artif Organs 1995; 19:1262-1268.

29 Kouidi E, Iacovides A, Iordanidis P, et al: Exercise renal rehabilitation program: Psychological effects. Nephron 1997;77:152-158.

- 30 Frey S, Mir AR, Lucas M: Visceral protein status and caloric intake in exercising versus nonexercising individuals with end-stage renal disease. J Renal Nutr 1999;9:71-77.

- 31 Deligiannis A, Kouidi E, Tourkantonis A: Effects of physical training on heart rate variability in patients on hemodialysis. Am J Cardiol 1999;84:197-202. 
-32 Deligiannis A, Kouidi E, Tassoulas E, Gigis P, Tourkantonis A, Coats A: Cardiac effects of exercise rehabilitation in hemodialysis patients. Int J Cardiol 1999;70:253-266.

$\checkmark 33$ DePaul V, Moreland J, Eager T, Clase CM: The effectiveness of aerobic and muscle strength training in patients receiving hemodialysis and EPO: a randomized controlled trial. Am J Kidney Dis 2002;40:1219-1229.

-34 Painter P, Moore GE, Carlson L, et al: Effects of exercise training plus normalization of hematocrit on exercise capacity and health-related quality of life. Am J Kidney Dis 2002;39: 257-265.

-35 Konstantinidou E, Koukouvou G, Kouidi E, Deligiannis A, Tourkantonis A: Exercise training in patients with end-stage renal disease on hemodialysis: comparison of three rehabilitation programs. J Rehabil Med 2002;34:4045.

36 Kouidi E, Grekas D, Deligiannis A, Tourkantonis A: Outcomes of long-term exercise training in dialysis patients: comparison of two training methods. Clin Nephrol 2004;61:S31S38.
37 Molsted S, Eidemak I, Sorensen HT, Kristensen JH: Five months of physical exercise in hemodialysis patients: effects on aerobic capacity, physical function and self-rated health. Nephron Clin Pract 2004;96:76-81.

38 Parsons T, Toffelmire E, King-VanVlack C: The effect of an exercise program during hemodialysis on dialysis efficacy, blood pressure and quality of life in end-stage renal disease (ESRD) patients. Clin Nephrol 2004;61:261-274.

39 American College of Sports Medicine Position Stand: The recommended quantity and quality of exercise for developing and maintaining cardiorespiratory and muscular fitness, and flexibility in healthy adults. Med Sci Sports Exerc 1998;30:975-991.

40 Marrades RM, Roca J, Campistol JM, et al: Effects of erythropoietin on muscle $\mathrm{O}_{2}$ transport during exercise in patients with chronic renal failure. J Clin Invest 1996;97:20922100.

41 Kong C, Tattersall J, Greenwood R, Farrington $\mathrm{K}$ : The effect of exercise during hemodialysis on solute removal. Nephrol Dial Transplant 1999; 14:2927-2931.

42 Fiatarone Singh MA: Exercise comes of age: rationale and recommendations for a geriatric exercise prescription. J Gerontol 2002;57A: M262-M282.
43 Beck A, Ward C, Mendelson M, Mock J, Erbaugh J: An inventory for measuring depression. Arch Gen Psychiatry 1961;4:561-571.

44 Stewart AL, Ware JEJ: Measuring Functioning and Well-being: The Medical Outcomes Study Approach. Durham, Duke University Press, 1992.

45 Spitzer W, Dobson A, Hall J, Chesterman E, Levi J, Shepherd R, Battista R, Catchlove B: Measuring the quality of life of cancer patients: a concise QL-Index for use by physicians. J Chron Dis 1981;14:585-597.

$46 \mathrm{~K} / \mathrm{DOQI}$ Clinical Practice Guidelines for Cardiovascular Disease in Dialysis Patients. Am J Kidney Dis 2005;45:S16-S153.

$\checkmark 47$ Moher D, Schulz K, Altman D: The CONSORT statement: Revised recommendations for improving the quality of reports of parallel group randomised trials. Lancet 2001;357: 1191-1194.

48 Garibotto G: Muscle amino acid metabolism and the control of muscle protein turnover in patients with chronic renal failure. Nutrition 1999; 15:145-155. 